\title{
VIDEO SIGNAL TRANSMISSION WITH ADAPTIVE CODING PROCEDURE BASED ON MULTIAGENT MODEL
}

\author{
Stanislav Tomashevich \\ Faculty of Control Systems and Robotics, ITMO University, \\ Russia \\ tomashevich.stanislav@gmail.com
}

Article history:

Received 11.07.2019, Accepted 22.09.2019

\begin{abstract}
The paper proposes the use of an adaptive coding algorithm for processing and transmitting video signals. Encoding process of video signals intend to divide the color of each pixel into three components (red, green and blue). The whole video frame could be represented as networked system where each pixel is a independent agent. In this case, agents are understood as a system having any dynamics. Thus, the movement of a pixel is described by a change in the intensity of its RGB components. This approach allows us to consider the process of intensity change as the trajectory of the dynamic system and apply observation and coding methods to it. The proposed approach allows to encode signals in real time without their prior complete analysis. Depending on the coding rate and the nature of the signal, it is possible to save the amount of information transmitted as a result of the algorithm. Observer is used to get estimation of colors signals and multiagent model is used to get to accurate estimation process.
\end{abstract}

\section{Key words}

Coding, video signals, observer, multiagent system

\section{Introduction}

Recently, with the development of wireless communications, there are more and more opportunities for autonomous systems interacting with the environment, with other system and users. For this information exchange, it is necessary to establish a communication channel, which may have low bandwidth. Examples of the signals that can be transmitted between systems include navigation data, state vectors, various information received from sensors, including audio and video signals. In this regard, it becomes relevant to use such coding, which can process signals in real-time for subsequent transmission through a data channel with limited bandwidth.
The adaptive coding algorithm proposed for this problem is one of the algorithms that works in real-time. Most of the common algorithms use a completely known signal, thereby having the ability to analyze the features and nature of the encoding signal. This approach allows one to encode data with minimal losses [Jayant et al., 1993; Chen and Guillemot, 2009; Chen and Guillemot, 2010; Noll, 1997]. The similar method is described in [Zierhofer, 2000], in the future works it is planned to research possibility to apply this method to encoding video signal with multiagent approach and compare with method proposed in this paper.

The algorithm proposed in this paper is based on an algorithm using quantization with memory [Andrievsky et al., 2007; Fradkov et al., 2010], which, in turn, is based on the method proposed for the static encoder [Goodman and Gersho, 1974]. An alternative application of similar algorithms can be found, for example, in problems of coding navigation data of aircraft [Andrievsky and Fradkov, 2010; Andrievsky and Fradkov, 2014] or different areas connected with quantization and time samplings [Andrievsky and Orlov, 2019; Andrievsky, 2016].

The problem of transferring information from one quadrocopter to another was solved with this procedure. This issue is relevant with the increasing popularity of multi-agent systems (see, for example, [Fax and Murray, 2004; Proskurnikov and Fradkov, 2016; Parsheva and Ternovaja, 2019]). Such systems forms to a group of agents that communicate with each other through a communication channel (see [Tomashevich and Belyavsky, 2017; Andrievskiy et al., 2015; Fradkov et al., 2016]).

In classic approaches observers are used to get estimations for different signals and its derivatives, e.g. [Ahmed et al., 2015; Avelar et al., 2018; Furtat and Nekhoroshikh, 2017a; Furtat and Nekhoroshikh, 2017b]. However, in the present paper observer is used for recovering the sampled signal on the receiver. Such approach helps to decrease amount of information transferring over any digital channel. 
Thus, due to the similar nature of video signals, it is possible to apply adaptive coding methods to encode frames changing in real-time. This paper focuses on the ways and results of this application.

\section{Adaptive coding procedure}

The following section presents the principle of the adaptive coding procedure for encoding and decoding the information contained in the signal [1]. Let $y[k]$ be the scalar signal to be transmitted over the digital communication channel at discrete points in time $t_{k}=k T$, where $T$ is the discrete interval, $k$ is the step number. With binary encoding, the relationship $R=T^{-1}$ holds, where $R$ is the data transfer rate, expressed in bits per second. Let us use the binary static quantizer:

$$
q(\sigma, M)=M[k] \operatorname{sign}(\sigma),
$$

where $M[k]$ is the quantizer level, $\sigma[k]=$ $\operatorname{sign}(y[k]-\hat{y}[k])$, where $\hat{y}[k]$ is the signal estimation, which will be formed below. The $\sigma$ value is represented by a one-bit character of the coding alphabet. It is essential to assume that the encoder and decoder work synchronously, and also that the data transmission in the communication channel is lossless. As an algorithm for tuning the quantization level, we use the algorithm that is different from that proposed in [Andrievsky and Fradkov, 2010; Andrievsky and Fradkov, 2014]:

$$
\begin{aligned}
& \lambda[k]=(s[k]+s[k-1]+s[k-2]) / 3, \\
& M[k+1]=m+ \begin{cases}\rho M[k], & \text { if }|\lambda[k]| \leq 0.5, \\
M[k] / \rho, & \text { otherwise, }\end{cases} \\
& \lambda[0]=\lambda[1]=0, \quad M[0]=M_{0},
\end{aligned}
$$

To estimate the encoded signal, an observer of the following form is introduced into the encoder and decoder:

$$
\left\{\begin{array}{l}
\hat{y}[k+1]=\hat{y}[k]+T \hat{V}[k]+l_{1} \sigma[k], \\
\hat{V}[k+1]=\hat{V}[k]+l_{2} \sigma[k],
\end{array}\right.
$$

where $\hat{V}[k]$ is the the estimate of signals velocity at instants $t_{k} ; l_{1}, l_{2}$ are observer parameters the components of the observer gain vector $L$.

The parameter $\lambda[k]$ characterizes the changes scatter in the encoded signal, which makes it possible to determine the desired direction of parameter $M[k]$ changing. The quantization range increases if the encoded signal changes rapidly, and vice versa, decreases with small changes in this signal. This behavior allows coder to evaluate the new encoding value and then restore it on the decoder. The parameter $m$ defines the minimum level of quantization. The decoder, receiving values, adjusts the quantization level similarly by the algorithm (2) by calculating the current value (1), which is calculated as $q[k]=y[k]-\hat{y}[k]$, the decoder uses the restored value $y[k]$ to estimate the transmitted signal using an observer (3), which model emulate the dynamics of the encoded signal.

\section{Video coding method}

To encode a video signal, it is proposed to divide the entire image into pixels, each of which is described in three colors red, green and blue. For the representation of each color, one octet is used, the values of which are denoted for convenience by integers from 0 to 255 inclusive, where 0 is the minimum and 255 is the maximum intensity. The sequence of video frames for each pixel determines the process of changing the intensities of each of the color components. Apply the algorithm described above to each color in a pixel for all image pixels. Obviously, for a video signal without an abrupt frame change, the process of changing the intensity of the three colors can be slowly changing, which will allow it to be estimated more precise. Thus, the coding of a pixel while one discretization step is performed with three bits. To encode a full image without compression and estimation, one needs to spend 24 bits per pixel. Obviously, the size of the encoded signal will always be less than the original, up to a high coding frequency more than eight times than the sampling rate of the original video signal.

To demonstrate the process of encoding a video signal, we use an arbitrary video fragment converted into a set of frames. The coding results for the color components of one of the pixels are shown in Fig. 1. Fig. 2 shows estimation process for the whole arbitrary image.
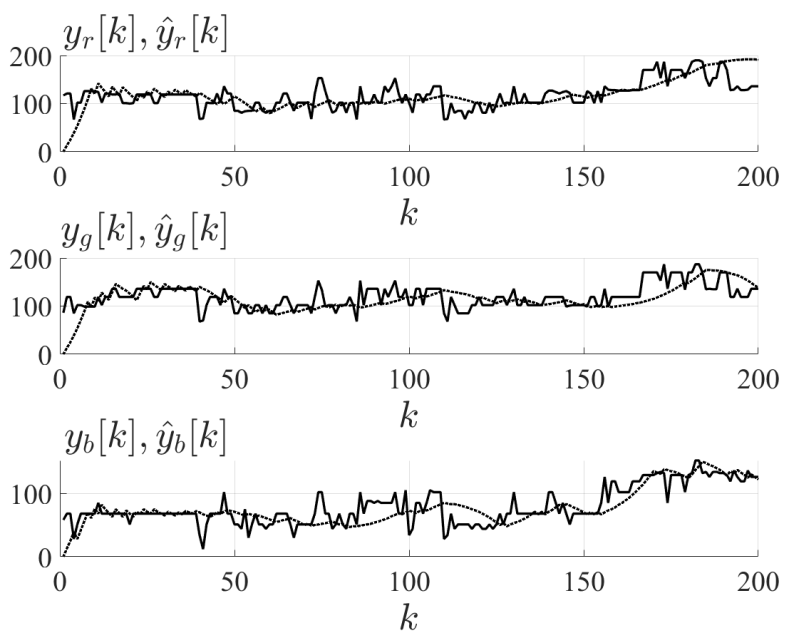

Figure 1. Time histories of RGB components one of the pixels and its estimations.

\section{Multiagent approach}

Based on idea that the video signal is some real set of frames (not just alternation of different pixels), pixels time-history is close to the time-histories of adjacent pixels. In this case the whole system could be presented as set of agents which are connected with only its adjacent as it shown at the Fig. 3. 

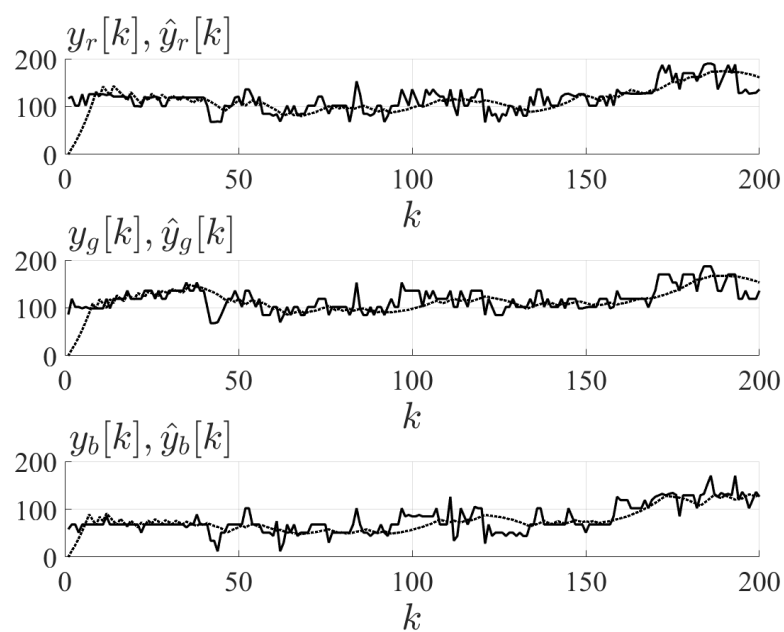

Figure 4. Time histories of RGB components one of the pixels and its estimations for multi-agent structure.

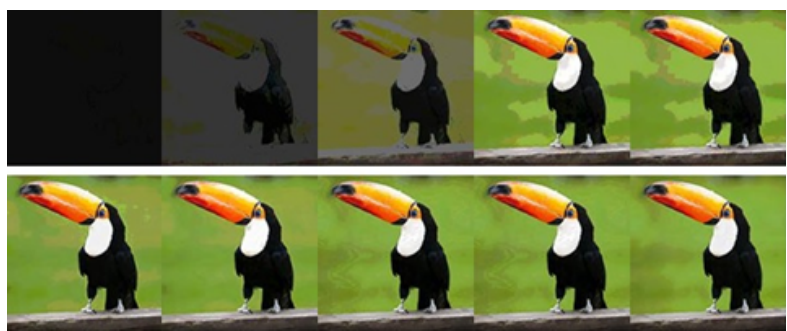

Figure 2. Several steps of static image estimation.

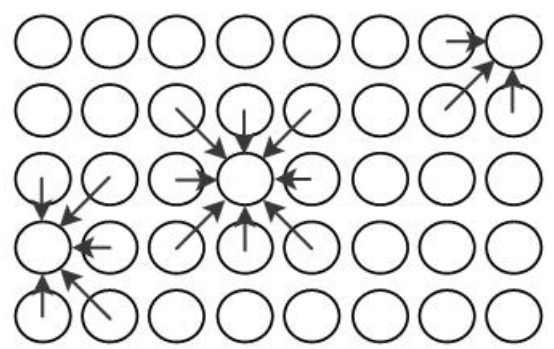

Figure 3. Influence diagram of adjacent pixels.

In this system it is considered that signal for every pixel (except pixels on the frame border) will be generated as

$$
\hat{y}_{i, j}[k+1]=\left(\sum_{a=i-i}^{i+1} \sum_{b=j-1}^{j+1} y_{a, b}[k]\right) \bar{\alpha},
$$

where $\bar{\alpha}=\alpha$ for $a=i, b=j$ and $\bar{\alpha}$ for other cases, $\alpha$ - trust coefficient, describing how current signal is true. In another words, it is a weight for the certain pixel, $\hat{y}[k]$ - corrected signal.

Procedure of choosing $\alpha$ could be taken as follows:

$$
\alpha= \begin{cases}\alpha_{1}, & \text { if } y_{i, j}[k+1]-y_{i, j}[k]>\kappa, \\ \alpha_{2}, & \text { else, }\end{cases}
$$

where $\kappa, \alpha_{1}, \alpha_{2}$ - some parameter, choosing by designer.

In the Fig. 4 time histories for multi-agent case for every pixel are shown.

Multi-agent approach was performed with taking $\alpha_{1}=$ $0.6, \alpha_{2}=0.8$ and $\kappa=100$. Results of the both approaches were obtained for the same pixel in the same video file. Let us use quality criteria in the following way:

$$
q=\frac{1}{k_{e}} \sum_{k=1}^{200} \sqrt{e_{r}^{2}[k]+e_{g}^{2}[k]+e_{b}^{2}[k]} .
$$

where $e_{r}[k]=\hat{y}_{r}[k]-y_{r}[k], e_{g}=\hat{y}_{g}[k]-y_{g}[k]$ and $e_{b}[k]=\hat{y}_{b}[k]-y_{b}[k]$ respectively, $k_{e}-$ number of steps. Average estimation error in the instance $k$ could be derived as $\bar{e}[k]=\sqrt{e_{r}^{2}[k]+e_{g}^{2}[k]+e_{b}^{2}[k]}$.

Following results were obtained based on timehistories in the Fig. 1 and 4: $q_{1}=34.15$ for the common approach and $q_{2}=31.24$ for the multi-agent approach. Estimation errors for both approaches are shown in the Fig. 5. One could see that the second method has the better quality based on the quality criteria (6), describing coding error for the time interval. The difference is almost $9 \%$.

Moreover, it is important to compare amount of transmitted information in case of non-adaptive coding. As it was discussed earlier there is no pre-processing procedure in proposed method, so comparison could be done with pixel by pixel transmission. BMP color standard has the same method to store pixels: TrueColor uses only 24 bit for one pixel. For an video signal with size $n \times m$ pixels and with duration $t$, the overall information transmitted via channel equals $n \times m \times t \times 24$ bits. With the proposed method one could reduce overall information by three times to $n \times m \times t \times 3$ as every pixel could be codded with one bit.

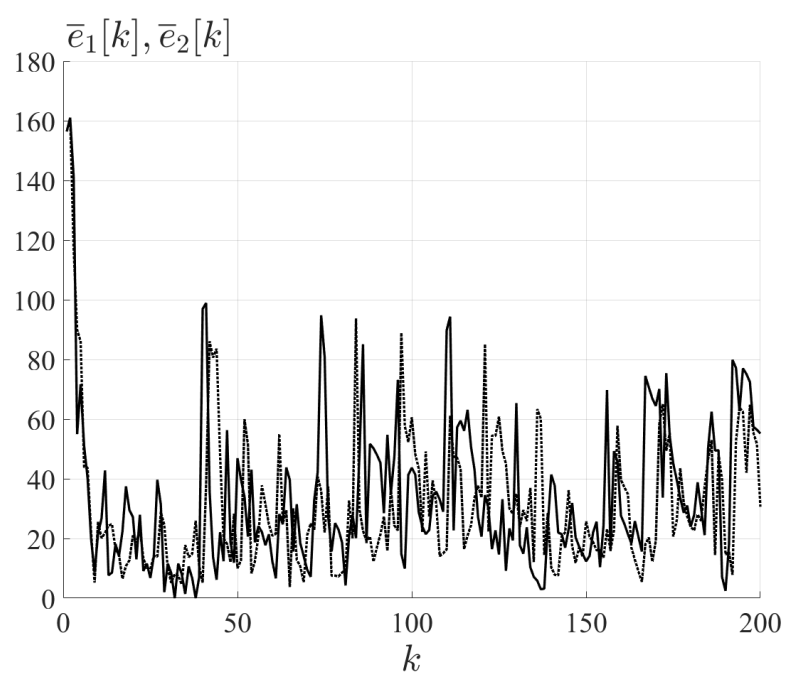

Figure 5. Time histories of estimation errors $\bar{e}_{1}[k]$ and $\bar{e}_{2}[k]$. 


\section{Conclusion}

This paper describes the applying of an adaptive coding procedure for video signals in case of limited bandwidth of the communication channel. The proposed method allows real-time systems to use low coding rate with loss of quality, subjectively imperceptible and unimportant information. Simple approach was presented also as multi-agent based method. The effectiveness of the proposed methods is demonstrated by the example of encoding an arbitrary video signal. In the future, it is possible to improve the quality of coding of signals by choosing a different model that describes the dynamics of the encoded signals more precise. Thus, one of the research ways is the study of the nature of the video signals dynamics and the corresponding modification of the proposed algorithm.

\section{Acknowledgements}

Designing the observer for multiagent approach supported by RFBR, grant 18-38-20037, multiagent approach for coding procedure supported by RFBR, grant 17-08-01728.

\section{References}

Ahmed, H., Ushirobira, R., Efimov, D., Tran, D., and Massabuau, J.-C. (2015). Velocity estimation of valve movement in oysters forwater quality surveillance. IFAC-PapersOnLine, 48 (11), pp. 333-338.

Andrievskiy, B., Tomashevich, S., Fradkov, A. L., and Amelin, K. (2015). Quadrocopters formation control over the limited-band communication network. IFACPapersOnLine.

Andrievsky, B. (2016). Numerical evaluation of controlled synchronization for chaotic Chua systems over the limited-band data erasure channel. Cybernetics and Physics, 5(2), pp. 43-51.

Andrievsky, B. and Fradkov, A. (2010). Adaptive coding for position estimation in formation flight control. Proc. IFAC Workshop Adaptation and Learning in Control and Signal Processing (ALCOSP 2010), pp. 72-76.

Andrievsky, B., Fradkov, A., and Peaucelle, D. (2007). State estimation over the limited-band communication channel for pitch motion control of LAAS helicopter benchmark. Proc. 17th IFAC Symp. Aut. Contr. Aerospace (ACA 2007), Toulouse, France.

Andrievsky, B. and Fradkov, A. L. (2014). Adaptive coding for maneuvering UAV tracking over the digital communication channel. International Congress on Ultra Modern Telecommunications and Control Systems and Workshops, ICUMT 2014, St. Petersburg, Russia, pp. 236-241.

Andrievsky, B. and Orlov, Y. (2019). Numerical evaluation of sine-gordon chain energy control via subdomains state feedback under quantization and time sampling. Cybernetics and Physics, 8(1), pp. 18-28.
Avelar, A., Salgado, I., Ahmed, H., Mera, M., and Chairez, I. (2018). Differential neural networks observer for second order systems with sampled and quantized output. IFAC-PapersOnLine, 51 (13), pp. 490-495.

Chen, Z. and Guillemot, C. (2009). Perceptuallyfriendly H.264/AVC video coding. Image Processing (ICIP), 2009 16th IEEE International Conference on., pp. 3417-3420.

Chen, Z. and Guillemot, C. (2010). Perceptuallyfriendly H.264/AVC video coding based on foveated just-noticeable-distortion model. Transactions on Circuits and Systems for Video Technology, 20(6), pp. 806-819.

Fax, J. A. and Murray, R. M. (2004). Information flow and cooperative control of vehicle formations. IEEE Trans. Automat. Contr., 49 (9), pp. 1465-1476.

Fradkov, A., Andrievskiy, B., and Evans, R. (2010). Hybrid quantized observer for multi-input-multi-output nonlinear systems. Dynamics and control of hybrid mechanical systems. World Scientific Series on Nonlinear Science, Series B, 14, pp. 89-102.

Fradkov, A. L., Tomashevich, S., Andrievskiy, B., Amelin, K., and Kaliteevskiy, I. (2016). Adaptive coding for data exchange between quadrotors in the formation. IFAC-PapersOnLine, 49(13), pp. 275-280.

Furtat, I. and Nekhoroshikh, A. (2017a). Robust algorithm using delay for multi-agent systems. UBS, $\mathbf{6 5}$, pp. 41-59.

Furtat, I. and Nekhoroshikh, A. (2017b). Robust stabilization of linear plants under uncertainties and highfrequency measurement noises. Proc. 25th Mediterranean Conference on Control and Automation. Valletta, Malta, pp. 1275-1280.

Goodman, D. and Gersho, A. (1974). Theory of an adaptive quantizer. IEEE Transactions on Communications, 22 (8), pp. 1037-1045.

Jayant, N., Johnston, J., and Safranek, R. (1993). Signal compression based on models of human perception. Proceedings of the IEEE, 81 (10), pp. 1385-1422.

Noll, P. (1997). MPEG digital audio coding. IEEE Signal Processing Magazine, 20 (6), pp. 806-819.

Parsheva, E. and Ternovaja, G. (2019). Robust control for multiply connected systems with state delay. $C y$ bernetics and Phisics, 8(1), pp. 38-44.

Proskurnikov, A. V. and Fradkov, A. L. (2016). Problems and methods of network control. Automation and Remote Control, 77 (10), pp. 1711-1740.

Tomashevich, S. and Belyavsky, A. (2017). Navigation data transfer in a quadrotor formation via a binary communication channel with adaptive coding and data erasure. 24th Saint Petersburg International Conference on Integrated Navigation Systems (ICINS), pp. 1-3.

Zierhofer, C. M. (2000). Adaptive sigma-delta modulation with one-bit quantization. IEEE Transactions on Circuits and Systems II: Analog and Digital Signal Processing, 47 (5), pp. 408-415. 